\title{
Joint modeling versus discriminant analysis for dynamic prediction of a binary outcome based on longitudinal data: A simulation study
}

Rana Dandis ( $\square$ rana.dandis@radboudumc.nl )

Radboudumc https://orcid.org/0000-0003-4002-1386

Joanna IntHout

Radboudumc

Kit Roes

Radboudumc

Steven Teerenstra

Radboudumc

Research article

Keywords: Joint model, Longitudinal discriminant analysis, Dynamic prediction, longitudinal data, dichotomous outcome

Posted Date: September 8th, 2020

DOI: https://doi.org/10.21203/rs.3.rs-59503/v2

License: (c) (i) This work is licensed under a Creative Commons Attribution 4.0 International License. Read Full License 
The authors have withdrawn this preprint from Research Square 